\title{
Avaliação do risco cardiovascular por meio do índice LAP em pacientes não obesas com síndrome dos ovários policísticos
}

\author{
Evaluation of cardiovascular risk by the LAP index in \\ non-obese patients with polycystic ovary syndrome
}

1 Programa de Pós-Graduação em Ciências da Saúde, Universidade Federal do Rio Grande do Norte (UFRN), Natal, RN, Brasil ${ }^{2}$ Departamento de Ginecologia e Obstetrícia, Maternidade Escola Januário Cicco, UFRN, Natal, RN, Brasil

${ }^{3}$ Departamento de Análises Clínicas e Toxicológicas, Centro de Ciências da Saúde, UFRN, Natal, RN, Brasil ${ }^{4}$ Departamento de Tocoginecologia, UFRN, Natal, RN, Brasi ${ }^{5}$ Departamento de Morfologia, Centro de Biociências, UFRN, Natal, RN, Brasi

\author{
Correspondência para: \\ George Dantas Azevedo \\ Departamento de Morfologia, \\ Centro de Biociências, \\ Campus Universitário UFRN, \\ Lagoa Nova \\ 59078-970 - Natal, RN, Brasil \\ georgedantas@uol.com.br \\ Recebido em 15/Maio/2010 \\ Aceito em 14/Ago/2010
}

\begin{abstract}
Eduardo Caldas Costa', Joceline Cássia Ferezini de Sá', Elvira Maria Mafaldo Soares², Telma Maria Araújo Moura Lemos ${ }^{3}$, Técia Maria de Oliveira Maranhão4, George Dantas Azevedo ${ }^{1,5}$
\end{abstract}

\begin{abstract}
RESUMO
Objetivo: Analisar o risco cardiovascular de mulheres não obesas com síndrome dos ovários policísticos (SOP) por meio do índice LAP (lipid accumulation product). Sujeitos e métodos: 283 pacientes (18-34 anos) alocadas do Hospital Universitário da Universidade Federal do Rio Grande do Norte, Natal-RN, foram divididas em quatro grupos: 1 ) SOP magras ( $n=35)$; 2 ) saudáveis magras ( $n=162)$; 3) SOP com sobrepeso $(n=28)$; 4) saudáveis com sobrepeso $(n=58)$. Resultados: Pacientes com SOP apresentaram valores superiores do índice LAP em comparação às saudáveis: magras $(22,26$ vs. $15,87 \mathrm{~cm} . \mathrm{mmol} / \mathrm{L} ; \mathrm{p}=0,007)$; sobrepeso $(40,83 \mathrm{vs}$. 26,32 $\mathrm{cm} . \mathrm{mmol} / \mathrm{L} ; \mathrm{p}=0,001)$. O percentual de mulheres acima do percentil 75 do índice LAP também foi superior nos subgrupos com SOP: magras $(17,1$ vs. $6,8 \% ; p=0,04)$; sobrepeso $(37,5$ vs. $13,8 \% ; p=0,01)$. Conclusões: Na amostra estudada, as pacientes com SOP apresentaram maior risco cardiovascular avaliado pelo índice LAP, quando comparadas a mulheres saudáveis, mesmo na ausência de obesidade. Arq Bras Endocrinol Metab. 2010;54(7):630-5
\end{abstract}

\section{Descritores}

Síndrome dos ovários policísticos; obesidade central; dislipidemia; resistência à insulina; fatores de risco; doenças cardiovasculares

\begin{abstract}
Objective: To analyze the cardiovascular risk of non-obese women with polycystic ovary syndrome (PCOS) by the LAP index (lipid accumulation product). Subjects and methods: 283 patients (18-34 years) assigned at the University Hospital of the Federal University of Rio Grande do Norte, Natal-RN, were divided into four groups: 1) lean PCOS $(n=35) ; 2$ ) healthy lean ( $n=$ 162); 3) overweight PCOS ( $n=28) ; 4$ ) healthy overweight ( $n=58)$. Results: Patients with PCOS showed higher values of the LAP index than healthy controls: lean $(22.26 \mathrm{vs} .15 .87 \mathrm{~cm} . \mathrm{mmol} / \mathrm{L}$; $p=0.007)$; overweight (40.83 vs. $26.32 \mathrm{~cm} . \mathrm{mmol} / \mathrm{L} ; \mathrm{p}=0.001)$. The percentage of women above the 75th percentile of the LAP index was also higher in the subgroups with PCOS: lean (17.1 vs. $6.8 \% ; p=0.04$ ); overweight ( 37.5 vs. $13.8 \% ; p=0.01$ ). Conclusions: In our sample, patients with PCOS showed higher cardiovascular risk assessed by the LAP index in relation to healthy women, even in the absence of obesity. Arq Bras Endocrinol Metab. 2010;54(7):630-5
\end{abstract}

\section{Keywords}

Polycystic ovary syndrome; central obesity; dyslipidemia; insulin resistance; risk factors; cardiovascular diseases 
Além das características específicas que definem a síndrome, a SOP é reconhecida por estar associada a um conjunto de fatores de risco para doença cardiovascular (DCV), destacando-se dislipidemia (3), diabetes tipo 2 (4), obesidade central (5) e síndrome metabólica (6), com a participação da resistência à insulina (RI) como agente de integração dessas diversas anormalidades cardiometabólicas (7).

Entre os fatores de risco cardiovascular comuns na SOP, a obesidade central tem merecido destacada atenção $(5,8-14)$, em virtude do fato de que a distribuição visceral de gordura está relacionada com a RI $(13,14)$, aspecto intimamente associado à fisiopatologia da SOP (15). Além disso, parece não haver dúvidas quanto à relação entre marcadores de obesidade do tipo androide e fatores de risco relacionados ao sistema cardiovascular nesse subgrupo da população feminina $(5,8,11,12,14)$.

Sendo a obesidade uma característica comum na SOP (16), é habitual na literatura estudos relacionados à avaliação do risco cardiovascular envolvendo mulheres obesas (índice de massa corporal $\geq 30 \mathrm{~kg} / \mathrm{m}^{2}$ ). Tal fato pode gerar um viés importante na interpretação clínica dos resultados, já que a obesidade per se é fator de risco independente para DCV (17). Diante disso, no que diz respeito à investigação de marcadores de risco cardiovascular na SOP, torna-se importante a realização de estudos envolvendo exclusivamente mulheres não obesas.

Nesse sentido, o índice LAP (lipid accumulation product), "ferramenta clínica" simples que combina uma medida antropométrica (circunferência da cintura) e outra bioquímica (concentração de triglicerídeos), pode ser de grande valia. Sua determinação em mulheres é dada pela seguinte equação: (circunferência da cintura $[\mathrm{cm}]-58) \times($ triglicerídeos $[\mathrm{mmol} / \mathrm{L}])(18)$. Além da facilidade para mensuração e do baixo custo, o índice LAP tem se destacado como importante indicador de risco cardiovascular em mulheres (18), inclusive com SOP (19).

Portanto, diante da escassez de trabalhos com foco investigativo na avaliação do risco cardiovascular de mulheres não obesas com SOP, o objetivo do presente estudo foi analisar o risco cardiovascular desse subgrupo da população feminina por meio do índice LAP e compará-las com mulheres hígidas.

\section{SUJEITOS E MÉTODOS}

Por meio de estudo transversal, pacientes não obesas $\left(\right.$ IMC $\left.<30 \mathrm{~kg} / \mathrm{m}^{2}\right)$, não fumantes, alocadas no Am- bulatório de Ginecologia Endócrina da Maternidade Escola Januário Cicco (MEJC), Universidade Federal do Rio Grande do Norte (UFRN), Natal-RN, foram avaliadas no que diz respeito a medidas antropométricas, clínicas e bioquímicas.

Todas as pacientes foram submetidas a exame clínico constando de medida da massa corporal $(\mathrm{kg})$, estatura (m) e circunferência da cintura $(\mathrm{cm})$. A circunferência da cintura foi mensurada no ponto médio entre a última costela e a crista ilíaca. Amostras de sangue venoso foram coletadas entre $08 \mathrm{~h} 00 \mathrm{~min}$ e $10 \mathrm{~h} 00 \mathrm{~min}$, após jejum prévio de 12 horas. A glicose sérica foi medida pelo método glicose oxidase. Os níveis de colesterol total, HDL-colesterol e triglicerídeos foram determinados por ensaio colorimétrico (BioSystems, Barcelona, Espanha). O nível de LDL-colesterol foi calculado usando a fórmula de Friedewald (colesterol total - [HDL-colesterol + triglicerídeos / 5]). O índice LAP foi determinado pela equação descrita por Kahn (18): (circunferência da cintura $[\mathrm{cm}]-58) \mathrm{x}($ triglicerídeos $[\mathrm{mmol} / \mathrm{L}])$.

Foram selecionadas para o estudo mulheres jovens com idade entre 18 e 34 anos. O diagnóstico de SOP foi confirmado mediante os critérios de Rotterdam (2). A exclusão de outras doenças, como hiperplasia adrenal congênita forma não clássica, disfunção de tireoide, hiperprolactinemia e diabetes melito, também foi considerada em todas as pacientes, por meio da realização de dosagens hormonais (17-hidroxiprogesterona, TSH, T4 livre e prolactina) e do teste oral de tolerância à glicose, de acordo com o protocolo do serviço. Outros critérios de exclusão para esse estudo foram: disfunção renal, disfunção hepática, uso de medicação com potencial para afetar a função reprodutiva ou metabólica, tais como contraceptivos orais, drogas antiandrogênicas, antilipêmicas, hipoglicemiantes orais, estatinas ou terapia com glicocorticoides, até 60 dias antes de ingressarem no estudo. Pacientes etilistas também foram excluídas da pesquisa.

Para análise comparativa no que diz respeito ao índice LAP, as 283 pacientes alocadas para o estudo foram divididas em quatro grupos: 1 ) SOP magras ( $\mathrm{n}=$ $35) ; 2)$ saudáveis magras $(\mathrm{n}=162) ; 3)$ SOP com sobrepeso $(\mathrm{n}=28) ; 4)$ saudáveis com sobrepeso $(\mathrm{n}=58)$. A categorização dos grupos foi realizada pelo índice de massa corporal (IMC), de acordo com a Organização Mundial da Saúde (OMS) (20) - magra: IMC = 18,5$24,9 \mathrm{~kg} / \mathrm{m}^{2}$; sobrepeso: $\mathrm{IMC}=25-29,9 \mathrm{~kg} / \mathrm{m}^{2}$.

O estudo foi aprovado pelo Comitê de Ética em Pesquisa da UFRN (protocolo número 126/04) e todas as 
participantes assinaram termo de consentimento livre e esclarecido (TCLE), conforme resolução 196/96 do Conselho Nacional de Saúde.

\section{Análise estatística}

Os dados apresentaram distribuição normal, avaliados pelo método Kolmogorov-Smirnov. Os resultados estão expressos em média, desvio-padrão da média, frequência absoluta e relativa. Os testes t de Student para amostras independentes e qui-quadrado foram utilizados para análise de diferença entre os grupos. O pacote estatístico SPSS ${ }^{\circledR}$ versão 17.0 para Windows (SPSS, Inc., Chicago IL) foi utilizado para esses fins. Em todos os casos, foi considerado estatisticamente significativo um valor de $\mathrm{p}<0,05$.

Recorrendo-se a cálculos de poder estatístico a posteriori, verificou-se que o dimensionamento amostral utilizado permitiria detectar diferenças estatisticamente significativas entre os grupos com nível de confiança de 95\% e poder estatístico entre 75\% (grupo magras) e 90\% (grupo com sobrepeso). Para tal, foi utilizado o software GraphPad StatMate versão 1.0li (San Diego CA, USA).

\section{RESULTADOS}

Na tabela 1, estão descritos os dados antropométricos e bioquímicos referentes aos grupos de mulheres magras e com sobrepeso - SOP e controle. O grupo SOP apresentou valores superiores de circunferência da cintura e glicose de jejum, e menor valor de HDL-colesterol em relação ao grupo controle, independente do IMC $(\mathrm{p}<0,05)$. Nas pacientes com sobrepeso, o grupo SOP ainda apresentou maior média de colesterol total, LDLcolesterol e triglicerídeos, quando comparado ao controle $(\mathrm{p}<0,05)$.
$\mathrm{Na}$ figura 1, encontra-se a análise comparativa do índice LAP entre os grupos. As pacientes com SOP apresentaram valores superiores do índice LAP, em comparação ao grupo controle, tanto no subgrupo de mulheres magras $(22,26 \pm 18,89$ vs. $15,87 \pm 10,39$ $\mathrm{cm} . \mathrm{mmol} / \mathrm{L}$, respectivamente; $\mathrm{p}=0,007)$, quanto naquelas com sobrepeso $(40,83 \pm 20,86$ vs. $26,32 \pm 15,74$ $\mathrm{cm} . \mathrm{mmol} / \mathrm{L}$, respectivamente; $\mathrm{p}=0,001)$.

A figura 2 apresenta a análise de frequência dos subgrupos na faixa acima do percentil 75 do índice LAP (18), de acordo com a faixa etária. Tanto no subgrupo de mulheres magras $(17,1$ vs. $6,8 \%$, respectivamente; $\mathrm{p}=0,04)$ quanto com sobrepeso $(37,5$ vs. $13,8 \%$, respectivamente; $\mathrm{p}=0,01$ ) houve maior distribuição de pacientes com SOP na zona do índice LAP supracitada, em comparação com as mulheres saudáveis.
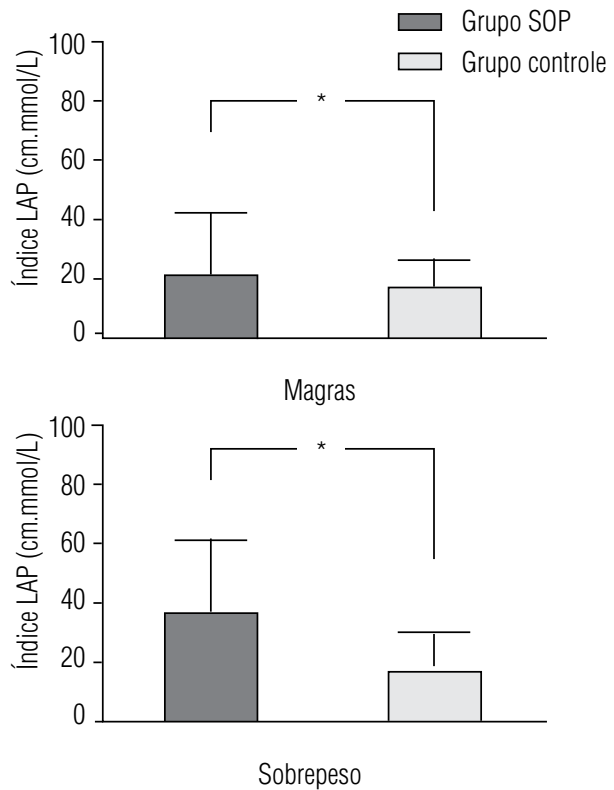

Figura 1. Análise do índice LAP: grupo SOP vs. grupo controle.

* Diferença estatisticamente significativa entre os grupos (teste $t$ de Student para amostras independentes $-p<0,05$ )

Tabela 1. Parâmetros antropométricos e bioquímicos da amostra do estudo: grupo SOP vs. controle

\begin{tabular}{|c|c|c|c|c|}
\hline \multirow{2}{*}{ Variáveis } & \multicolumn{2}{|c|}{ Magras $\left(\right.$ IMC $\left.<25 \mathrm{~kg} / \mathrm{m}^{2}\right)$} & \multicolumn{2}{|c|}{ Sobrepeso (IMC 25-29,9 kg/m²) } \\
\hline & SOP & Controle & SOP & Controle \\
\hline Idade (anos) & $24,4 \pm 4,7$ & $25,6 \pm 4,4$ & $26,8 \pm 4,2$ & $27,3 \pm 3,9$ \\
\hline IMC (kg/m²) & $22,1 \pm 2,2$ & $21,7 \pm 2,1$ & $27,6 \pm 1,5$ & $26,9 \pm 1,5$ \\
\hline Cintura (cm) & $74,9 \pm 6,4^{*}$ & $72,6 \pm 5,7$ & $88,5 \pm 8,2^{*}$ & $82,5 \pm 6,1$ \\
\hline Colesterol total (mg/dL) & $169,9 \pm 32,8$ & $171,3 \pm 44,4$ & $197,1 \pm 43,4^{*}$ & $177,7 \pm 41,1$ \\
\hline Glicose de jejum (mg/dL) & $81,9 \pm 8,9^{*}$ & $76,4 \pm 10,5$ & $83,5 \pm 14,5^{\star}$ & $76,3 \pm 13,3$ \\
\hline LDL-colesterol (mg/dL) & $104,2 \pm 33,5$ & $97,9 \pm 46,8$ & $132,2 \pm 36,2^{*}$ & $103,8 \pm 47,5$ \\
\hline HDL-colesterol (mg/dL) & $44,5 \pm 11,4^{*}$ & $53,6 \pm 17,5$ & $40,0 \pm 10,8^{*}$ & $54,9 \pm 23,4$ \\
\hline Triglicerídeos (mg/dL) & $105,7 \pm 61,1$ & $98,6 \pm 53,0$ & $125,0 \pm 71,5^{\star}$ & $94,9 \pm 46,1$ \\
\hline
\end{tabular}

IMC: índice de massa corporal; * diferença estatisticamente significativa em relação ao grupo controle (Teste t de Student para amostras independentes - $p<0,05$ ). 

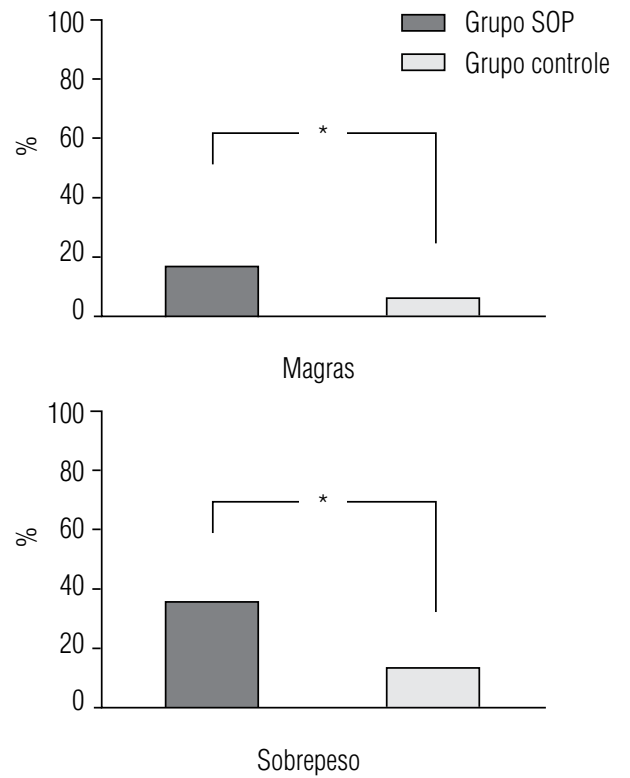

Figura 2. Prevalência de mulheres acima do percentil 75 do índice LAP, determinado por Kahn (18): grupo SOP vs. grupo controle.

* Diferença estatisticamente significativa entre os grupos (teste qui-quadrado $-p<0,05$ ).

\section{DISCUSSÃO}

O objetivo do presente estudo foi analisar o risco cardiovascular de pacientes não obesas com SOP por meio do índice LAP e compará-las com mulheres hígidas. Pelo fato de as mulheres com SOP apresentarem maior quantidade de gordura abdominal e perfil lipídico desfavorável em relação às mulheres hígidas (5,10,21-25), é de se esperar que elas apresentem valores superiores para o índice LAP, o que foi confirmado na presente pesquisa, mesmo no subgrupo de mulheres magras. É importante destacar que esse é o primeiro trabalho com foco investigativo na avaliação do risco cardiovascular de mulheres brasileiras não obesas com SOP pelo índice LAP.

Não há dúvidas de que mulheres com SOP apresentam elevada prevalência de obesidade central $(5,8,9$ $11,13,14)$. Estudos apontam que 50\% a $60 \%$ dessas pacientes apresentam obesidade abdominal, independente do IMC $(21,22)$. Tal fato se torna ainda mais relevante se considerarmos que a gordura centralizada foi o fator de risco cardiovascular que mais respondeu pelos casos de infarto agudo do miocárdio (IAM) nas mulheres sul-americanas (risco atribuível à população = 63\%), de acordo com o Inter-Heart Study (23).

No tocante a composição corporal, Kirchengast e Huber (22) demonstraram que mulheres magras com SOP apresentam maior quantidade de gordura corporal e menor quantidade de massa magra, quando pareadas com mulheres hígidas em relação a idade, massa corpo- ral e IMC. Nesse mesmo estudo, os autores observaram ainda que todas as mulheres hígidas apresentaram distribuição ginecoide de gordura e, em $30 \%$ das mulheres com SOP, houve distribuição androide. Esses dados corroboram os resultados do presente estudo, haja vista que, mesmo pareando pelo IMC, as pacientes com SOP analisadas apresentaram circunferência da cintura superior em relação aos subgrupos constituídos por mulheres saudáveis.

Por apresentar considerável prevalência em mulheres na idade reprodutiva e frequente associação com situações clínicas diversas, a SOP tem merecido atenção especial em decorrência das potenciais repercussões sobre a saúde da mulher. Nesse sentido, na atualidade os objetivos do tratamento para SOP não se restringem somente à abordagem das repercussões reprodutivas, como infertilidade, anovulação e hirsutismo. A avaliação, prevenção e/ou tratamento da saúde cardiovascular também devem fazer parte desse contexto terapêutico (24).

Recentemente, Costa e cols. (12) verificaram, em mulheres jovens com SOP $(26,5$ anos $-\mathrm{n}=102)$, alta prevalência de fatores de risco cardiovascular, principalmente relacionados ao perfil lipídico (colesterol total $\geq 200 \mathrm{mg} / \mathrm{dL}=31,4 \%$; HDL-colesterol $<50 \mathrm{mg} / \mathrm{dL}$ $=76,5 \%$; LDL-colesterol $\geq 160 \mathrm{mg} / \mathrm{dL}=29,4 \%$; triglicerídeos $\geq 150 \mathrm{mg} / \mathrm{dL}=32,4 \%$ ). Na presente pesquisa, apenas as pacientes com SOP do subgrupo com sobrepeso apresentaram um perfil lipídico desfavorável em relação ao grupo controle, conforme apresentado na tabela 1. É possível especular que tal fato ocorreu pela existência de uma tríade comum na SOP que potencializa alterações referentes ao perfil lipídico: excesso de gordura, RI e hiperandrogenismo (3). Isso pode explicar, em parte, o que aponta o NCEP ATP-III ( $\mathrm{Na-}$ tional Cholesterol Education Program) (25): 70\% das pacientes com SOP exibem anormalidades na concentração de lipídios plasmáticos.

$\mathrm{Na}$ perspectiva atual de avaliação do risco cardiovascular em mulheres jovens com SOP (24), torna-se relevante a inclusão e análise mais específica do subgrupo de mulheres magras, diante da carência de trabalhos nessa linha de investigação. Logo, nossos resultados referentes ao índice LAP tornam-se mais relevantes pela exclusão do viés da obesidade, o que sugere que a SOP possa exercer um papel considerável no que diz respeito ao aumento do risco cardiovascular. Os mecanismos para tal ainda não estão completamente esclarecidos, podendo ser atribuídos ao hiperandrogenismo, resis- 
tência periférica à insulina e maior deposição central de gordura, fatores esses comumente presentes na síndrome, inclusive no subgrupo de mulheres magras.

É importante destacar que o índice LAP representa um importante indicador não só de risco cardiovascular $(18,19)$, mas também de RI (19), diabetes tipo 2 (26) e mortalidade (27). Dentro dessa perspectiva, por meio da combinação de duas medidas simples e de baixo custo (circunferência da cintura e concentração plasmática de triglicerídeos), o índice LAP pode ser utilizado na prática clínica para avaliar e monitorar o risco cardiovascular de mulheres jovens com SOP. Outros marcadores de risco cardiovascular, como o Score de Framingham (28) e o Reynold Risk Score (29), necessitam de uma quantidade maior de variáveis envolvidas para determinação do risco cardiovascular, acarretando custos e tecnologias adicionais.

Wiltgen e cols. (19) avaliaram o índice LAP em mulheres com SOP (média de 26,6 anos e $29,5 \mathrm{~kg} / \mathrm{m}^{2}$ de IMC) e verificaram valor de $37,87 \mathrm{~cm} . \mathrm{mmol} / \mathrm{L}$, sendo muito semelhante aos nossos achados no subgrupo com sobrepeso $(40,83 \mathrm{~cm} . \mathrm{mmol} / \mathrm{L})$. Ainda corroborando com o presente estudo, os autores observaram diferença entre mulheres com SOP e saudáveis (pacientes ovulatórias sem hirsutismo). Entretanto, os pesquisadores citados não realizaram análise dicotômica referente ao índice LAP em diferentes classificações do IMC.

Em 2005, Kahn (18), analisando os dados do NHANES III (Third National Health and Nutrition Examination Survey), determinou percentis (25) do índice LAP, de acordo com sexo e faixa etária. Ao avaliar a distribuição de mulheres acima do percentil 75 , nossos dados apontam que as pacientes com SOP magras e com sobrepeso estão em maior prevalência nessa faixa de risco, quando comparadas aos respectivos grupos controles. Esse achado demonstra que há uma parcela considerável de pacientes não obesas com SOP situada em um patamar de risco cardiovascular significativo. Entretanto, é importante salientar que estudos complementares envolvendo mulheres magras com SOP (IMC $\left.<25 \mathrm{~kg} / \mathrm{m}^{2}\right)$ com maior tamanho amostral se fazem necessários para elucidar de forma definitiva os achados observados no presente estudo.

Diante dos achados obtidos, parece importante definir e empreender estratégias de caráter preventivo de forma precoce em pacientes não obesas com SOP, com o objetivo de evitar eventos mórbidos futuros relacionados ao sistema cardiovascular. Portanto, modificações no estilo de vida, incluindo prática regular de exercício físico e dieta saudável, devem fazer parte do contexto terapêutico dessas pacientes.
Agradecimentos: Ao Ministério da Saúde, à Secretaria de Estado da Saúde do Rio Grande do Norte, ao Conselho Nacional de Desenvolvimento Científico e Tecnológico $(\mathrm{CNPq})$ e à Fundação de Amparo à Pesquisa do Estado do Rio Grande do Norte (Fapern), pelo financiamento do presente estudo, por meio do Edital Pesquisa para o Sistema Único de Saúde (SUS).

Declaração: os autores declaram não haver conflitos de interesse científico neste estudo.

\section{REFERÊNCIAS}

1. Norman RJ, Dewailly D, Legro RS, Hickey TE. Polycystic ovary syndrome. Lancet. 2007;370(9588):685-97.

2. The Rotterdam ESHRE/ASRM-Sponsored PCOS consensus workshop group. Revised 2003 consensus on diagnostic criteria and long-term health risks related to polycystic ovary syndrome. Fertil Steril. 2004;81(1):19-25.

3. Diamanti-Kandarakis E, Papavassiliou AG, Kandarakis SA, Chrousos GP. Pathophysiology and types of dyslipidemia in PCOS. Trends Endocrinol Metab. 2007;18(7):280-5.

4. Cibula D, Cífková F, Fanta M, Poledne R, Zivny J, Skibová J. Increased risk of non-insulin dependent diabetes mellitus, arterial hypertension and coronary artery disease in perimenopausal women with a history of the polycystic ovary syndrome. Hum Reprod. 2000;15(4):785-9.

5. Cascella T, Palomba S, De Sio I, Manguso F, Giallauria F, De Simone $B$, et al. Visceral fat is associated with cardiovascular risk in women with polycystic ovary syndrome. Hum Reprod. 2008;23(1):153-9.

6. Soares EM, Azevedo GD, Gadelha RG, LemosTM, MaranhãoTM. Prevalence of the metabolic syndrome and its components in Brazilian women with polycystic ovary syndrome. Fertil Steril. 2008;89(3):649-55.

7. Teede HJ, Hutchison S, Zoungas S, Meyer C. Insulin resistance, the metabolic syndrome, diabetes, and cardiovascular disease risk in women with PCOS. Endocrine. 2006;30(1):45-53.

8. Carmina E, Bucchieri S, Esposito A, Del Puente A, Mansueto P, Orio $F$, et al. Abdominal fat quantity and distribution in women with polycystic ovary syndrome and extent of its relation to insulin resistance. J Clin Endocrinol Metab. 2007;92(7):2500-5.

9. Diamanti-Kandarakis E. Role of obesity and adiposity in polycystic ovary syndrome. Int J Obes (Lond). 2007;31(Suppl. 2):S8-S13.

10. Escobar-Morreale HF, San Millán JL. Abdominal obesity and the polycystic ovary syndrome. Trends Endocrinol Metab. 2007;18(7):266-72.

11. Oh JY, Sung YA, Lee HJ, Oh JY, Chung HW, Park H. Optimal waist circumference for prediction of metabolic syndrome in young Korean women with polycystic ovary syndrome. Obesity (Silver Spring). 2010;18(3):593-7.

12. Costa EC, Soares EMM, Lemos TMAM, MaranhãoTMO, Azevedo GD. Índices de obesidade central e fatores de risco cardiovascular na síndrome dos ovários policísticos. Arq Bras Cardiol. 2010;94(5):633-8.

13. Lord J, Thomas R, Fox B, Acharya U, Wilkin T. The central issue? Visceral fat mass is a good marker of insulin resistance and metabolic disturbance in women with polycystic ovary syndrome. BJOG. 2006;113(10):1203-9.

14. Puder JJ, Varga S, Kraenzlin M, De Geyter C, Keller U, Müller B. Central fat excess in polycystic ovary syndrome: relation with low-grade inflammation and insulin resistance. J Clin Endocrinol Metab. 2005;90(11):6014-21. 
15. Huber-Buchholz MM, Carey DG, Norman RJ. Restoration of reproductive potential by lifestyle modification in obese polycystic ovary syndrome: role of insulin sensitivity and luteinizing hormone. J Clin Endocrinol Metab. 1999;84(4):1470-4.

16. Vrbikova J, Hainer V. Obesity and polycystic ovary syndrome. Obes Facts. 2009;2(1):26-35.

17. Hubert HB, Feinleib M, McNamara PM, Castelli WP. Obesity as an independent risk factor for cardiovascular disease: a 26-year follow-up of participants in the Framingham Heart Study. Circulation. 1983;67:968-77.

18. Kahn HS. The "lipid accumulation" product performs better than the body mass index for recognizing cardiovascular risk: a population-based comparison. BMC Cardiovasc Disord. 2005;5:26.

19. Wiltgen D, Benedetto IG, Mastella LS, Spritzer PM. Lipid accumulation product index: a reliable marker of cardiovascular risk in polycystic ovary syndrome. Hum Reprod. 2009;24(7):1726-31.

20. World Health Organization. Obesity preventing and managing the global epidemic. Report of a WHO consultation on obesity. Geneva. 3-5 June, 1997.

21. Horejsi R, Moller R, Rackl S, Giuliani A, Freytag U, Crailsheim K, et al. Android subcutaneous adipose tissue topography in lean and obese women suffering from PCOS: comparison with type 2 diabetic women. Am J Phys Anthropol. 2004;124(3):275-81.

22. Kirchengast $S$, Huber J. Body composition characteristics and body fat distribution in lean women with polycystic ovary syndrome. Hum Reprod. 2001;16(6):1255-60.
23. Yusuf S, Hawken S, Ounpuu S, Dans T, Avezum A, Lanas F, et al. Effect of potentially modifiable risk factors associated with myocardial infarction in 52 countries (the INTERHEART study): casecontrol study. Lancet. 2004;364:937-52.

24. Wild RA, Carmina E, Diamanti-Kandarakis E, Dokras A, EscobarMorreale HF, Futterweit W, et al. Assessment of cardiovascular risk and prevention of cardiovascular disease in women with the polycystic ovary syndrome: a position statement by the Androgen Excess and Polycystic Ovary Syndrome (AE-PCOS) Society. J Clin Endocrinol Metab. 2010;95(5):2038-49.

25. Third Report of the National Cholesterol Education Program (NCEP) Expert Panel on Detection, Evaluation, and Treatment of High Blood Cholesterol in Adults (Adult Treatment Panel III) Final Report. Circulation. 2002;106:3143-421.

26. Kahn HS. The lipid accumulation product is better than BMI for identifying diabetes: a population-based comparison. Diabetes Care. 2006;29(1):151-3.

27. Ioachimescu AG, Brennan DM, Hoar BM, Hoogwerf BJ. The lipid accumulation product and all-cause mortality in patients at high cardiovascular risk: a PreCIS database study. Obesity (Silver Spring). 2010;18:1836-44.

28. Wilson PW, D'Agostino RB, Levy D, Belanger AM, Silbershatz $H$, Kannel WB. Prediction of coronary heart disease using risk factor categories. Circulation. 1998;97(18):1837-47.

29. Ridker PM, Buring JE, Rifai N, Cook NR. Development and validation of improved algorithms for the assessment of global cardiovascular risk in women: the Reynolds Risk Score. JAMA. 2007;297(6):611-9. 